\title{
Exact dynamics and squeezing in two harmonic modes coupled through angular momentum
}

\author{
N. Canosa ${ }^{1}$, Swapan Mandal ${ }^{1,2}$ and R. Rossignoli ${ }^{1}$ \\ ${ }^{1}$ Departamento de Física -IFLP, F.C.E., Universidad Nacional de La Plata, C. C. 67, \\ 1900 La Plata, Argentina \\ ${ }^{2}$ Department of Physics, Visva-Bharati, Santiniketan-731235, India
}

\begin{abstract}
We investigate the exact dynamics of a system of two independent harmonic oscillators coupled through their angular momentum. The exact analytic solution of the equations of motion for the field operators is derived, and the conditions for dynamical stability are obtained. As application, we examine the emergence of squeezing and mode entanglement for an arbitrary separable coherent initial state. It is shown that close to instability, the system develops considerable entanglement, which is accompanied with simultaneous squeezing in the coordinate of one oscillator and the momentum of the other oscillator. In contrast, for weak coupling away from instability, the generated entanglement is small, with weak alternating squeezing in the coordinate and momentum of each oscillator. Approximate expressions describing these regimes are also provided.
\end{abstract}


Exact dynamics and squeezing in two harmonic modes coupled through angular momentum2

\section{Introduction}

Models based on coupled harmonic oscillators have long attracted attention in several different fields due to their wide range of applications [1, 2, , 3, 4, 5, 6, 4, 8, 9, 10]. In particular, the case of two harmonic modes coupled through their angular momentum, which describes the motion of a charged particle within a general harmonic trap in a uniform magnetic field or, equivalently, the motion in a rotating anisotropic harmonic potential [11, 12, 13, has been employed in distinct scenarios, such as rotating nuclei [13], quantum dots in a magnetic field [14] and fast rotating Bose-Einstein condensates [15, 16, 17, 18] within the lowest Landau level approximation [19, 20, 21]. Since its Hamiltonian is quadratic in the field operators, the model is also suitable for simulation with optical techniques [22].

In a previous work [23] its dynamics in coordinate representation was analyzed in detail, showing that it exhibits a complex dynamical phase diagram, with stable as well as distinct types of unstable (i.e., unbounded) dynamics. We have also examined the generated entanglement between the modes, both in ground and thermal states

24] (vacuum and thermal entanglement) as well as that obtained after starting from a separable vacuum state [25]. It was shown, in particular, that this system is able to mimic typical entanglement growth regimes arising after a quantum quench in complex many body scenarios [26]. Entanglement is of course essential for quantum information applications [27], and a large entanglement growth with time after starting from a separable state in a many-body system, is indicative of a system dynamics which cannot be efficiently simulated by classical means.

On the other hand, quantum squeezing constitutes another topic of great current interest [28, 29, 30, 31]. Its relation with entanglement has been investigated in different systems [28, 29, 30, 31, 32, 33, 34], with entanglement normally inducing squeezing in certain observables. In particular, in [32] the exact dynamics of entanglement and squeezing in a two-mode Bose-Einstein condensate interacting through a Josephsonlike coupling was determined. Squeezing is important for quantum metrology, i.e., for improving the accuracy in quantum measurements [31, 35], and it has been shown that in some cases spin squeezing can be employed to detect entanglement [28, 30, 31, 33]. Nonetheless, in some regimes (like the linear case of the model considered in [32]) squeezing may also arise without substantial entanglement.

In this work we first derive the exact analytic expressions for the temporal evolution of the Heisenberg field operators of two harmonic modes coupled through their angular momentum. The obtained result is valid for all values of the system parameters, i.e., in stable as well as unstable dynamical regimes, and allows to determine the exact evolution of an arbitrary observable of the system. We then apply this result to determine and examine the dynamics of squeezing, which has so far not been investigated in this model, and its relation with the generated entanglement, when starting from a separable coherent initial state. We will show that different regimes can arise depending on the value of the rotational frequency. Close to the instability point, appreciable 
Exact dynamics and squeezing in two harmonic modes coupled through angular momentum3

entanglement is generated, accompanied with simultaneous squeezing in one of the variables of each mode, while for small couplings, the generated entanglement is weak, with small squeezing appearing in both variables of the mode at alternating times. Approximate simple expressions describing these two distinct regimes are also provided.

\section{Formalism}

\subsection{The model}

The Hamiltonian of the system under study can be written as

$$
H=\frac{1}{2 m} P_{1}^{2}+\frac{m \omega_{1}^{2}}{2} Q_{1}^{2}+\frac{1}{2 m} P_{2}^{2}+\frac{m \omega_{2}^{2}}{2} Q_{2}^{2}-\omega\left(Q_{1} P_{2}-P_{1} Q_{2}\right),
$$

where the subscripts 1 and 2 refer obviously to the first and second oscillator. The oscillator frequencies $\omega_{1}, \omega_{2}$ and the rotation frequency $\omega$ will be assumed real and satisfying, without loss of generality,

$$
\omega_{1} \geq \omega_{2}>0, \quad \omega \geq 0 .
$$

In the case of a particle of charge $e$ in a magnetic field $\boldsymbol{H}$ along the $z$ axis within a harmonic trap with spring constants $K_{1}, K_{2}$ in the $x, y$ plane, we have $\omega=e|\boldsymbol{H}| /(2 m c)$, with $\omega_{j}^{2}=K_{j} / m+\omega^{2}(j=1,2)$ [23] (motion along $z$ is obviously decoupled from that in $x, y$ plane, which is that described by (11)). Eq. (11) also represents the intrinsic Hamiltonian describing the motion (in the $x, y$ plane) of a particle in a harmonic trap with constants $m \omega_{j}^{2}$ rotating with frequency $\omega$ around the $z$ axis [23].

By expressing the position $\left(Q_{j}\right)$ and momentum $\left(P_{j}\right)$ operators in terms of the dimensionless annihilation $\left(a_{j}\right)$ and creation $\left(a_{j}^{\dagger}\right)$ boson operators, $Q_{j}=\sqrt{\frac{\hbar}{2 m \omega_{j}}}\left(a_{j}+a_{j}^{\dagger}\right)$, $P_{j}=-i \sqrt{\frac{\hbar m \omega_{j}}{2}}\left(a_{j}-a_{j}^{\dagger}\right), j=1,2$, we can rewrite (1) as

$$
\begin{aligned}
H= & \hbar \omega_{1}\left(a_{1}^{\dagger} a_{1}+\frac{1}{2}\right)+\hbar \omega_{2}\left(a_{2}^{\dagger} a_{2}+\frac{1}{2}\right)-i \hbar \lambda_{+}\left(a_{2}^{\dagger} a_{1}-a_{1}^{\dagger} a_{2}\right) \\
& -i \hbar \lambda_{-}\left(a_{1} a_{2}-a_{1}^{\dagger} a_{2}^{\dagger}\right),
\end{aligned}
$$

where

$$
\lambda_{ \pm}=\omega\left(\frac{\omega_{1} \pm \omega_{2}}{2 \sqrt{\omega_{1} \omega_{2}}}\right) .
$$

In the isotropic case $\omega_{1}=\omega_{2}=\omega_{0}, \lambda_{-}=0$ and both $H$ and the angular momentum, which becomes just $-i \hbar \lambda_{+}\left(a_{2}^{\dagger} a_{1}-a_{1}^{\dagger} a_{2}\right)$, commute with the total boson number $N=$ $a_{1}^{\dagger} a_{1}+a_{2}^{\dagger} a_{2}$ and between themselves.

However, in the anisotropic case $\omega_{1} \neq \omega_{2}$, the angular momentum term does not commute with $H$ and no longer conserves the total boson number. This entails, in particular, that in contrast with the isotropic case, the system vacuum will become entangled as $\omega$ increases, and that an initially separable state $\left|0_{1}\right\rangle\left|0_{2}\right\rangle$ (product of the vacuum of each oscillator) will become entangled as time increases if $\omega \neq 0$. In addition, the system may become unstable if $\omega$ increases sufficiently, as discussed below [23]. Note 
Exact dynamics and squeezing in two harmonic modes coupled through angular momentum4

that the exact dynamics of the special case $\omega_{1}=\omega_{2}$ with a $Q_{1} Q_{2}$ coupling, which was studied in detail in [4, corresponds formally to the case $\lambda_{+}=\lambda_{-}$, after replacing $a_{1} \rightarrow i a_{1}$. Also, the two-mode Bose Einstein condensate model of [32] (which contains as well non-linear terms) would formally correspond in the linear case to $\lambda_{-}=0$ and a time-dependent $\lambda_{+}$.

\subsection{Exact solution}

Let us now derive the explicit solution of the Heisenberg equations of motion for the field operators,

$$
i \hbar \dot{a}_{j}=\left[a_{j}, H\right], \quad j=1,2 .
$$

Eq. (6) leads to the linear system

$$
\begin{aligned}
& \dot{a_{1}}=-i \omega_{1} a_{1}+\lambda_{+} a_{2}+\lambda_{-} a_{2}^{\dagger} \\
& \dot{a_{2}}=-i \omega_{2} a_{2}-\lambda_{+} a_{1}+\lambda_{-} a_{1}^{\dagger}
\end{aligned}
$$

which can be written in matrix form as

$$
i\left(\begin{array}{c}
\dot{\boldsymbol{a}} \\
\dot{\boldsymbol{a}^{\dagger}}
\end{array}\right)=\mathcal{H}\left(\begin{array}{c}
\boldsymbol{a} \\
\boldsymbol{a}^{\dagger}
\end{array}\right)
$$

where $\boldsymbol{a}=\left(\begin{array}{c}a_{1} \\ a_{2}\end{array}\right), \boldsymbol{a}^{\dagger}=\left(\begin{array}{c}a_{1}^{\dagger} \\ a_{2}^{\dagger}\end{array}\right)$ and $\mathcal{H}$ is the $4 \times 4$ non-hermitian matrix

$$
\mathcal{H}=\left(\begin{array}{cccc}
\omega_{1} & i \lambda_{+} & 0 & i \lambda_{-} \\
-i \lambda_{+} & \omega_{2} & i \lambda_{-} & 0 \\
0 & i \lambda_{-} & -\omega_{1} & i \lambda_{+} \\
i \lambda_{-} & 0 & -i \lambda_{+} & -\omega_{2}
\end{array}\right)
$$

The exact solution of Eq. (8) can be expressed as

$$
\left(\begin{array}{c}
\boldsymbol{a}(t) \\
\boldsymbol{a}^{\dagger}(t)
\end{array}\right)=\mathcal{U}(t)\left(\begin{array}{c}
\boldsymbol{a}(0) \\
\boldsymbol{a}^{\dagger}(0)
\end{array}\right)
$$

where

$$
\mathcal{U}(t)=\exp [-i \mathcal{H} t]=\left(\begin{array}{cc}
U(t) & V(t) \\
V^{*}(t) & U^{*}(t)
\end{array}\right)
$$

is a $4 \times 4$ matrix satisfying (II denotes the $2 \times 2$ identity matrix)

$$
\mathcal{U}(t) \mathcal{M U}^{\dagger}(t)=\mathcal{M}, \quad \mathcal{M}=\left(\begin{array}{cc}
\mathbb{I} & 0 \\
0 & -\mathbb{I}
\end{array}\right)
$$

since $\mathcal{M H}^{\dagger} \mathcal{M}=\mathcal{H}$. Eq. (12) ensures the preservation of the equal time commutation relations $\forall t$ :

$$
\left[a_{i}(t), a_{j}^{\dagger}(t)\right]=\left[U U^{\dagger}-V V^{\dagger}\right]_{i j}(t)=\delta_{i j}, \quad\left[a_{i}(t), a_{j}(t)\right]=\left[U V^{\mathrm{t}}-V U^{t}\right]_{i j}(t)=0,(13
$$

implying that Eq. (10) represents a proper time-dependent Bogoliubov transformation for the field operators. 
Exact dynamics and squeezing in two harmonic modes coupled through angular momentum5

Setting in what follows $a_{j}(0) \equiv a_{j}, a_{j}^{\dagger}(0) \equiv a_{j}^{\dagger}$, Eq. (10) leads explicitly to

$$
a_{j}(t)=U_{j 1}(t) a_{1}+U_{j 2}(t) a_{2}+V_{j 1}(t) a_{1}^{\dagger}+V_{j 2}(t) a_{2}^{\dagger},
$$

and the corresponding adjoint equations for $a_{j}^{\dagger}(t)$, where the elements $U_{j k}(t), V_{j k}(t)$ can be obtained from Eq. (111) through the diagonalization of $\mathcal{H}$ :

$$
\begin{aligned}
U_{j j}(t)= & \frac{1}{2}\left\{\left(1+\gamma_{j}\right) \cos \omega_{+} t+\left(1-\gamma_{j}\right) \cos \omega_{-} t\right. \\
& \left.-i \omega_{j}\left[\left(1+\delta_{j}\right) \frac{\sin \omega_{+} t}{\omega_{+}}+\left(1-\delta_{j}\right) \frac{\sin \omega_{-} t}{\omega_{-}}\right]\right\} \\
V_{j j}(t)= & i(-1)^{j+1} \frac{\omega_{1} \omega_{2} \lambda_{+} \lambda_{-}}{\omega_{j} \Delta}\left(\frac{\sin \omega_{+} t}{\omega_{+}}-\frac{\sin \omega_{-} t}{\omega_{-}}\right), \\
\left(\begin{array}{c}
U_{12}(t) \\
V_{12}(t)
\end{array}\right)= & \frac{\lambda_{ \pm}}{2}\left[\left(1+\frac{\left(\omega_{1} \pm \omega_{2}\right)^{2}}{2 \Delta}\right) \frac{\sin \omega_{+} t}{\omega_{+}}+\left(1-\frac{\left(\omega_{1} \pm \omega_{2}\right)^{2}}{2 \Delta}\right) \frac{\sin \omega_{-} t}{\omega_{-}}\right. \\
& \left.+i \frac{\omega_{1} \pm \omega_{2}}{\Delta}\left(\cos \omega_{+} t-\cos \omega_{-} t\right)\right] \\
U_{21}(t)= & -U_{12}(t), \quad V_{21}(t)=V_{12}^{*}(t),
\end{aligned}
$$

with

$$
\gamma_{j}=(-1)^{j+1} \frac{\omega_{1}^{2}-\omega_{2}^{2}}{2 \Delta}, \quad \delta_{j}=\gamma_{j}+\frac{\omega^{2}\left(2 \omega_{j}^{2}+\omega_{1}^{2}+\omega_{2}^{2}\right)}{2 \Delta \omega_{j}^{2}} .
$$

Here $\omega_{ \pm}$are the system eigenfrequencies, i.e., the eigenvalues of the matrix $\mathcal{H}$ (which are $\left.\pm \omega_{+}, \pm \omega_{-}\right)$, given by

$$
\omega_{ \pm}=\sqrt{\lambda_{+}^{2}-\lambda_{-}^{2}+\frac{\omega_{1}^{2}+\omega_{2}^{2}}{2} \pm \Delta}=\sqrt{\omega^{2}+\frac{\omega_{1}^{2}+\omega_{2}^{2}}{2} \pm \Delta},
$$

where

$$
\begin{aligned}
\Delta & =\sqrt{\lambda_{+}^{2}\left(\omega_{1}+\omega_{2}\right)^{2}+\left(\omega_{1}-\omega_{2}\right)^{2}\left[\frac{\left(\omega_{1}+\omega_{2}\right)^{2}}{4}-\lambda_{-}^{2}\right]} \\
& =\sqrt{\frac{\left(\omega_{1}^{2}-\omega_{2}^{2}\right)^{2}}{4}+2 \omega^{2}\left(\omega_{1}^{2}+\omega_{2}^{2}\right)} .
\end{aligned}
$$

It is verified that for $\omega=0\left(\lambda_{ \pm}=0\right), \Delta=\frac{\omega_{1}^{2}-\omega_{2}^{2}}{2}$ and hence $\omega_{+(-)}=\omega_{1(2)}$, leading to $U_{j k}(t)=\delta_{j k} e^{-i \omega_{j} t}, V_{j k}(t)=0$. The free evolution $a_{j}(t)=e^{-i \omega_{j} t} a_{j}$ is then recovered. Also, in the special case $\omega_{1}=\omega_{2}=\omega$ and $\lambda_{+}=\lambda_{-}=\kappa$ we recover the eigenfrequencies $\omega_{ \pm}=\sqrt{\omega^{2} \pm 2 \kappa \omega}$ of [4].

On the other hand, in the isotropic case $\omega_{1}=\omega_{2}=\omega_{0}$, we have $\lambda_{-}=0$, implying $\Delta=2 \omega \omega_{0}$ and

$$
\omega_{ \pm}=\omega_{0} \pm \omega, \quad V(t)=0, \quad\left(\omega_{1}=\omega_{2}=\omega_{0}\right)
$$

which leads finally to

$$
a_{1}(t)=e^{-i \omega_{0} t}\left(a_{1} \cos \omega t+a_{2} \sin \omega t\right), \quad a_{2}(t)=e^{-i \omega_{0} t}\left(-a_{1} \sin \omega t+a_{2} \cos \omega t\right) .
$$

This is equivalent to a beam-splitter type transformation of angle $\omega t$ of the field operators. In this case the angular momentum term commutes with $H$ and just rotates the field operators with angular frequency $\omega$. 
Exact dynamics and squeezing in two harmonic modes coupled through angular momentum6

The general solution (14)-(18) can be derived by many other methods. For instance, we may write the solution of Eq. (10) for each operator as

$$
a_{j}(t)=e^{i H t / \hbar} a_{j} e^{-i H t / \hbar}=a_{j}+\frac{i t}{\hbar}\left[H, a_{j}\right]+\frac{1}{2 !}\left(\frac{i t}{\hbar}\right)^{2}\left[H,\left[H, a_{j}\right]\right]+\ldots
$$

which leads immediately to the form (14) for $a_{j}(t)$. And insertion of a trial solution of the form (14) in (7) (i.e., the so called Sen-Mandal approach [36, 37]) leads to a linear system of first order differential equations for the coefficients $U_{j k}(t), V_{j k}(t)$, namely $i \dot{\mathcal{U}}=\mathcal{H} \mathcal{U}$, i.e.,

$$
i\left(\begin{array}{c}
\dot{\boldsymbol{U}}_{k} \\
\dot{\boldsymbol{V}}_{k}^{*}
\end{array}\right)=\mathcal{H}\left(\begin{array}{c}
\boldsymbol{U}_{k} \\
\boldsymbol{V}_{k}^{*}
\end{array}\right), \quad k=1,2
$$

where $\boldsymbol{U}_{k}=\left(\begin{array}{c}U_{1 k} \\ U_{2 k}\end{array}\right), \boldsymbol{V}_{k}=\left(\begin{array}{c}V_{1 k} \\ V_{2 k}\end{array}\right)$ are the $k^{\text {th }}$ column of $U, V$. Eq. (26) and the initial conditions $U_{j k}(0)=\delta_{j k}, V_{j k}(0)=0$ lead again to the solution (15)-(18). We finally notice that the system (7) leads, after successive derivation, to the fully decoupled quartic equations

$$
\dddot{a}_{j}+\left(\omega_{1}^{2}+\omega_{2}^{2}+2\left(\lambda_{+}^{2}-\lambda_{-}^{2}\right)\right) \ddot{a}_{j}+\left(\left(\lambda_{+}+\lambda_{-}\right)^{2}-\omega_{1} \omega_{2}\right)\left(\left(\lambda_{+}-\lambda_{-}\right)^{2}-\omega_{1} \omega_{2}\right) a_{j}=0(27
$$

for $j=1,2$, which lead at once to the eigenfrequencies (20) (after inserting a trial solution $a_{j}(t) \propto e^{i \alpha t}$ ) and again to the solution (15)-(18) after inserting the initial conditions for the operators and their derivatives. The matrix $\mathcal{U}(t)$, and hence all coefficients $U_{j k}(t), V_{j k}(t)$, also satisfy Eq. (27).

\subsection{Dynamical stability and normal mode decomposition}

In the general case, a close inspection of the eigenvalues (20) reveals that $\omega_{ \pm}$are both real and non-zero only if $\lambda_{+}+\lambda_{-}<\sqrt{\omega_{1} \omega_{2}}$ or $\lambda_{+}-\lambda_{-}>\sqrt{\omega_{1} \omega_{2}}$, i.e., if $\omega<\omega_{2}$ or $\omega>\omega_{1}$, which is equivalent to

$$
\left(\omega-\omega_{1}\right)\left(\omega-\omega_{2}\right)>0 .
$$

Dynamical stability (bounded quasiperiodic dynamics) is then ensured if Eq. (28) is satisfied. On the other hand, if $\lambda_{+}-\lambda_{-}<\sqrt{\omega_{1} \omega_{2}}<\lambda_{+}+\lambda_{-}$, i.e., $\omega_{2}<\omega<\omega_{1}$ or in general

$$
\left(\omega-\omega_{1}\right)\left(\omega-\omega_{2}\right)<0,
$$

$\omega_{+}$remains real but $\omega_{-}$becomes imaginary $\left(\omega_{-}=i\left|\omega_{-}\right|\right)$implying that the dynamics becomes unbounded. In this case we should just replace

$$
\frac{\sin \omega_{-} t}{\omega_{-}} \rightarrow \frac{\sinh \left|\omega_{-}\right| t}{\left|\omega_{-}\right|}, \cos \omega_{-} t \rightarrow \cosh \left|\omega_{-}\right| t
$$

in Eqs. (15)-(18), entailing that all operators "increase" (i.e., deviate from their initial values) exponentially with time. Nevertheless, Eq. (12) and hence the commutation relations (13) remain satisfied. 
Exact dynamics and squeezing in two harmonic modes coupled through angular momentum7

Finally, if $\omega=\omega_{2}$ or $\omega=\omega_{1}$, i.e., if

$$
\left(\omega-\omega_{1}\right)\left(\omega-\omega_{2}\right)=0,
$$

we obtain a critical regime where $\omega_{+}>0$ but $\omega_{-}=0$, in which case the matrix $\mathcal{H}$ becomes non-diagonalizable unless $\omega_{1}=\omega_{2}$ (Landau case). If $\omega_{-}=0$, we should just replace the corresponding expressions by their natural limits, i.e.,

$$
\frac{\sin \omega_{-} t}{\omega_{-}} \rightarrow t, \quad \cos \omega_{-} t \rightarrow 1
$$

in Eqs. (15)-(18) (the ensuing solution also follows from (11) after using the Jordan canonical form of $\mathcal{H}$ for $\omega_{-}=0$ [23]), entailing that the dynamics is again unbounded if $\omega_{1} \neq \omega_{2}$, with the deviation from the initial values increasing now linearly with time. Eqs. (12) are again preserved. In the Landau case $\omega=\omega_{1}=\omega_{2}$, the coefficients of the linearly increasing terms vanish and the dynamics is again bounded, given by Eq. (23) for $\omega=\omega_{0}$.

A standard normal mode decomposition of $H$ becomes feasible in the dynamically stable phases $\left(\omega_{ \pm}>0\right)$ [23]. In terms of the standard normal mode boson operators $b_{ \pm}, b_{ \pm}^{\dagger}$ given in the appendix, we may express (11) in the first dynamically stable sector $\omega<\omega_{2}$ as

$$
H=\hbar \omega_{+}\left(b_{+}^{\dagger} b_{+}+\frac{1}{2}\right)+\hbar \omega_{-}\left(b_{-}^{\dagger} b_{-}+\frac{1}{2}\right), \omega<\omega_{2}
$$

with $b_{ \pm}(t)=e^{-i \omega_{ \pm} t} b_{ \pm}(0)$, whereas in the second dynamically stable sector $\omega>\omega_{1}$ we have

$$
H=\hbar \omega_{+}\left(b_{+}^{\dagger} b_{+}+\frac{1}{2}\right)-\hbar \omega_{-}\left(b_{-}^{\dagger} b_{-}+\frac{1}{2}\right), \omega>\omega_{1},
$$

with $b_{-}(t)=e^{i \omega_{-} t} b_{-}(0)$ (and $\omega_{-}>0$ ). Eq. (34) entails that in this region, the system is no longer energetically stable.

\section{Application}

\subsection{Squeezing and entanglement}

We have now all the elements for investigating the evolution of distinct quantum properties of the system, such as entanglement and squeezing. We start by noting that the number operators for each mode are given by (here $j, k, l=1,2$ )

$$
\begin{aligned}
N_{j}(t) \equiv a_{j}^{\dagger}(t) a_{j}(t)= & \sum_{k, l}\left[U_{j k}^{*}(t) U_{j l}(t) a_{k}^{\dagger} a_{l}+V_{j k}^{*}(t) V_{j l}(t) a_{k} a_{l}^{\dagger}\right. \\
& \left.+U_{j k}^{*}(t) V_{j l}(t) a_{k}^{\dagger} a_{l}^{\dagger}+V_{j k}^{*}(t) U_{j l}(t) a_{k} a_{l}\right],
\end{aligned}
$$

indicating that they will acquire a non-zero average even if there are initially no bosons: If the system starts at the separable vacuum $|00\rangle \equiv\left|0_{1}\right\rangle\left|0_{2}\right\rangle$, where $a_{j}\left|0_{j}\right\rangle=0$, from Eqs. (35) and (15)-(18) we obtain, setting $\langle O\rangle_{0} \equiv\langle 00|O| 00\rangle$ and $j, k=1,2$,

$$
\left\langle N_{j}(t)\right\rangle_{0}=\sum_{k}\left|V_{j k}(t)\right|^{2}
$$


Exact dynamics and squeezing in two harmonic modes coupled through angular momentum8

$$
\begin{aligned}
= & \frac{\omega^{2}\left(\omega_{1}-\omega_{2}\right)^{2}}{16 \omega_{1} \omega_{2}}\left[\left|\sum_{\nu= \pm}\left(1+\nu \frac{\left(\omega_{1}-\omega_{2}\right)^{2}}{2 \Delta}\right) \frac{\sin \omega_{\nu} t}{\omega_{\nu}}+i \nu \frac{\left(\omega_{1}-\omega_{2}\right) \cos \omega_{\nu} t}{\Delta}\right|^{2}\right. \\
& \left.+\frac{\omega^{2}\left(\omega_{1}+\omega_{2}\right)^{2} \omega_{1} \omega_{2}}{\omega_{j}^{2}|\Delta|^{2}}\left|\frac{\sin \omega_{+} t}{\omega_{+}}-\frac{\sin \omega_{-} t}{\omega_{-}}\right|^{2}\right]
\end{aligned}
$$

which will be normally non-zero for $t>0$ unless $\omega_{1}=\omega_{2} .\left\langle N_{j}(t)\right\rangle$ is then proportional to $\left(\omega_{1}-\omega_{2}\right)^{2}$, and is larger in the mode with the lowest frequency $\omega_{j}$ (due to the last term in (37)).

If the initial state is instead a product $\left|\alpha_{1} \alpha_{2}\right\rangle \equiv\left|\alpha_{1}\right\rangle\left|\alpha_{2}\right\rangle$ of coherent states $\left|\alpha_{j}\right\rangle$ for each oscillator, with $a_{j}\left|\alpha_{j}\right\rangle=\alpha_{j}\left|\alpha_{j}\right\rangle$, the same expressions (36)-(37) remain valid for the corresponding covariance of the operators $a_{j}^{\dagger}(t), a_{j}(t)$ :

$$
\left\langle N_{j}(t)\right\rangle_{\alpha}-\left\langle a_{j}^{\dagger}(t)\right\rangle_{\alpha}\left\langle a_{j}(t)\right\rangle_{\alpha}=\left\langle N_{j}(t)\right\rangle_{0}=\sum_{k}\left|V_{j k}(t)\right|^{2}
$$

where $\langle O\rangle_{\alpha} \equiv\left\langle\alpha_{1} \alpha_{2}|O| \alpha_{1} \alpha_{2}\right\rangle$. Eq. (38) is then independent of $\alpha_{1}$ and $\alpha_{2}$.

Similarly, we may evaluate the coordinates and momenta fluctuations and their dimensionless ratios to their initial values,

$$
R_{Q_{j}}^{2}(t)=\frac{\left\langle Q_{j}^{2}(t)\right\rangle_{\alpha}-\left\langle Q_{j}(t)\right\rangle_{\alpha}^{2}}{\left\langle Q_{j}^{2}(0)\right\rangle_{\alpha}-\left\langle Q_{j}(0)\right\rangle_{\alpha}^{2}}, \quad R_{P_{j}}^{2}(t)=\frac{\left\langle P_{j}^{2}(t)\right\rangle_{\alpha}-\left\langle P_{j}(t)\right\rangle_{\alpha}^{2}}{\left\langle P_{j}^{2}(0)\right\rangle_{\alpha}-\left\langle P_{j}(0)\right\rangle_{\alpha}^{2}},
$$

which for a coherent initial state satisfy $R_{Q_{j}}(t) R_{P_{j}}(t) \geq 1$, due to the uncertainty principle and the fact that a coherent initial state has minimum uncertainty. Squeezing in $Q_{j}$ or $P_{j}$ occurs whenever $R_{Q_{j}}(t)$ or $R_{P_{j}}(t)$ ) becomes smaller than 1 . We obtain, explicitly,

$$
\begin{aligned}
R_{Q_{j}\left(P_{j}\right)}^{2}(t) & =1+2\left[\left\langle N_{j}(t)\right\rangle_{\alpha}-\left|\left\langle a_{j}(t)\right\rangle_{\alpha}\right|^{2} \pm \operatorname{Re}\left(\left\langle a_{j}^{2}(t)\right\rangle_{\alpha}-\left\langle a_{j}(t)\right\rangle_{\alpha}^{2}\right)\right] \\
& =1+2\left[\left\langle N_{j}(t)\right\rangle_{0} \pm \operatorname{Re}\left(\left\langle a_{j}^{2}(t)\right\rangle_{0}\right)\right]
\end{aligned}
$$

where Re denotes real part, $+(-)$ corresponds to $Q_{j}\left(P_{j}\right)$ and

$$
\left\langle a_{j}^{2}(t)\right\rangle_{0}=\sum_{k} U_{j k}(t) V_{j k}(t) .
$$

These ratios are then also independent of $\alpha_{1}, \alpha_{2}$, and deviate from 1 unless $V(t)=0$.

An initial coherent state is a pure separable gaussian state, which under the present Hamiltonian will remain gaussian (but no longer separable) $\forall t$. Its entanglement entropy $S(t)$ can then be evaluated through the gaussian state formalism [24, 25, 38, 39, 40] and can be written as

$$
S(t)=-\operatorname{Tr} \rho_{j}(t) \ln \rho_{j}(t)=-f(t) \ln f(t)+[1+f(t)] \ln [1+f(t)],
$$

where $\rho_{j}(t)$ denotes the reduced state of one of the modes and $f(t)$ is the symplectic eigenvalue of the single mode covariance matrix:

$$
\begin{aligned}
f(t) & =\sqrt{\left(\left\langle N_{j}(t)\right\rangle_{\alpha}-\left|\left\langle a_{j}(t)\right\rangle_{\alpha}\right|^{2}+\frac{1}{2}\right)^{2}-\left|\left\langle a_{j}^{2}(t)\right\rangle_{\alpha}-\left\langle a_{j}(t)\right\rangle_{\alpha}^{2}\right|^{2}}-\frac{1}{2} \\
& =\sqrt{\left(\left\langle N_{j}(t)\right\rangle_{0}+\frac{1}{2}\right)^{2}-\left|\left\langle a_{j}^{2}(t)\right\rangle_{0}\right|^{2}}-\frac{1}{2}
\end{aligned}
$$


which is non-negative and the same for $j=1$ or $j=2$ if the state is pure and gaussian. It represents the effective occupation number of the mode [25]. It is obviously also independent of $\alpha_{1}, \alpha_{2}$, i.e., the same for any coherent initial state. The entanglement entropy (42) is just an increasing concave function of $f(t)$. Again, in the isotropic case $\omega_{1}=\omega_{2}, V(t)=0$ (Eq. (23) ), entailing no generated entanglement when starting from $\left|\alpha_{1} \alpha_{2}\right\rangle$.

\subsection{Results}
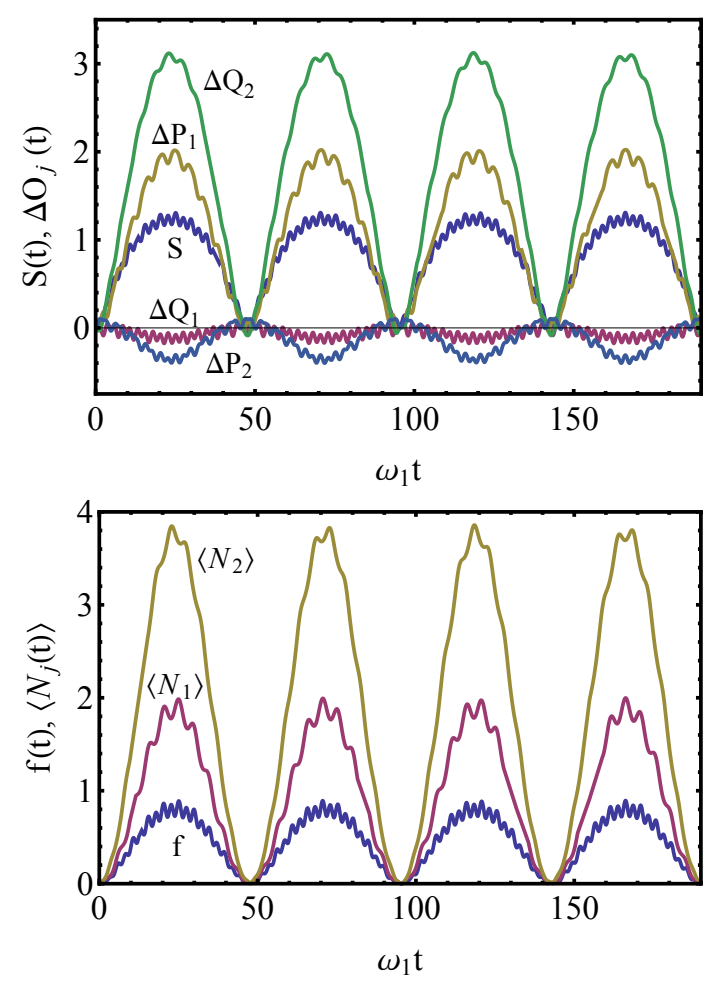

Figure 1. (Color online) Top: Temporal evolution of the entanglement entropy $S(t)$ (42) and the shifted squeezing ratios (44) for the operators $Q_{j}, P_{j}$ of each oscillator, starting from a separable coherent state. We have set here $\omega_{2}=\omega_{1} / 2$ with a rotation frequency $\omega=0.49 \omega_{1}$, such that the system is close to the first instability (occurring when $\omega$ reaches $\omega_{2}$ ). Both $Q_{1}$ and $P_{2}$ exhibit appreciable squeezing $\left(\Delta O_{j}(t)<0\right)$, whose evolution is in phase with that of entanglement. Bottom: The corresponding symplectic eigenvalue $f(t)$ (43) determining the entanglement entropy, and the average boson numbers of each mode when starting from the separable vacuum (or the covariances (38) when starting from a coherent state). Quantities plotted are dimensionless.

Results for the previous quantities are depicted in Figs. 12 for $\omega_{2}<\omega_{1}$. We concentrate on the first dynamically stable sector $\omega<\omega_{2}$. For improved visualization of squeezing, we use there the quantities

$$
\Delta Q_{j}(t) \equiv R_{Q_{j}}(t)-1, \Delta P_{j}(t) \equiv R_{P_{j}}(t)-1,
$$

with squeezing in $Q_{j}\left(P_{j}\right)$ indicated by a negative value of $\Delta Q_{j}\left(\Delta P_{j}\right)$. 
Exact dynamics and squeezing in two harmonic modes coupled through angular momentum10

In Fig. 1 we consider the anisotropic case $\omega_{2}=\omega_{1} / 2$ with $\omega$ close to $\omega_{2}$, i.e., to the first instability, such that $\omega_{+} \approx 1.31 \omega_{1}, \omega_{-} \approx 0.07 \omega_{1}$. The evolution then exhibits large amplitude low frequency oscillations governed by $\omega_{-}$, together with small amplitude high frequency oscillations governed by $\omega_{+}$. The picture clearly shows that in this regime squeezing and entanglement oscillate in phase: Maximum entanglement occurs at times $t_{n} \approx n \pi /\left(2 \omega_{-}\right), n$ odd (see Eqs. (45)-(46)), simultaneously with maximum squeezing in the operators $Q_{1}$ and $P_{2}$, and maximum average boson number in the oscillators (or maximum covariance (38) in the case of an initially coherent state).

These results can be approximately described by conserving just the main terms in $V_{j k}(t)$ and $U_{j k}(t)$ (Eqs. (15) $-(18)$ ) for small $\omega_{-}$, which are those proportional to $\omega_{-}^{-1}$. We obtain

$$
\begin{aligned}
& \left\langle N_{j}(t)\right\rangle_{0} \approx \frac{\omega^{2}\left(\omega_{1}-\omega_{2}\right)^{2}}{16 \omega_{1} \omega_{2}}\left[\left(1-\frac{\left(\omega_{1}-\omega_{2}\right)^{2}}{2 \Delta}\right)^{2}+\frac{\omega^{2}\left(\omega_{1}+\omega_{2}\right)^{2} \omega_{1} \omega_{2}}{\omega_{j}^{2} \Delta^{2}}\right] \frac{\sin ^{2} \omega_{-} t}{\omega_{-}^{2}} \\
& \left\langle a_{j}^{2}(t)\right\rangle_{0} \approx(-1)^{j} \frac{\omega^{2}\left(\omega_{1}^{2}-\omega_{2}^{2}\right)}{16 \omega_{1} \omega_{2}}\left[\frac{2 \omega_{1} \omega_{2}\left(1-\delta_{j}\right)}{\Delta}-\left(1-\frac{\left(\omega_{1}-\omega_{2}\right)^{2}}{2 \Delta}\right)\left(1-\frac{\left(\omega_{1}+\omega_{2}\right)^{2}}{2 \Delta}\right)\right] \frac{\sin ^{2} \omega_{-} t}{\omega_{-}^{2}}
\end{aligned}
$$

where $\delta_{j}$ is given in (19). Hence, both $\left\langle N_{j}(t)\right\rangle_{0}$ and $\left\langle a_{j}^{2}(t)\right\rangle_{0}$ become proportional to $\sin ^{2} \omega_{-} t$, entailing that all quantities plotted in Fig. 1 will be governed by this term, thus oscillating in phase. The presence of a factor $\omega_{j}^{-2}$ in (45) also entails $\left\langle N_{2}(t)\right\rangle_{0} \geq\left\langle N_{1}(t)\right\rangle_{0}$, i.e., the boson number will be larger in the mode with the smallest frequency, as verified in the bottom panel of Fig. 1,

Moreover, for $\omega$ below but close to $\omega_{2}$, the bracket in (46) will be positive for $j=1,2$ and larger for $j=2$ (since $0<\delta_{2}<\delta_{1}<1$ ), leading to $\left\langle a_{1}^{2}(t)\right\rangle_{0} \leq 0$ and $\left\langle a_{2}^{2}(t)\right\rangle_{0} \geq 0$, with $\left|\left\langle a_{1}^{2}(t)\right\rangle_{0}\right| \leq\left|\left\langle a_{2}^{2}(t)\right\rangle_{0}\right|$. Hence, according to Eq. (40), squeezing will occur in $Q_{1}$ and $P_{2}$, as seen in Fig. 1, being more pronounced in $P_{2}$. Eqs. (45)-(46) also show that squeezing and entanglement are driven by the anisotropy, i.e., they vanish for $\omega_{1}=\omega_{2}$ and at fixed $\omega$ become larger as $\omega_{1}-\omega_{2}$ increases (or the ratio $\omega_{2} / \omega_{1}$ decreases). They also increase, of course, as $\omega$ approaches $\omega_{2}$, i.e., as the first instability is reached $\left(\omega_{-}=0\right.$ for $\left.\omega=\omega_{2}\right)$. We also mention that for $\omega$ above but close to $\omega_{1}$ (i.e., in the second stable region but close to instability), the behavior is similar although squeezing will occur for $P_{1}$ and $Q_{2}$, since the bracket in (46) becomes negative (with $\delta_{2}>\delta_{1}>1$ ), implying $\left\langle a_{1}^{2}(t)\right\rangle_{0} \geq 0,\left\langle a_{2}^{2}(t)\right\rangle_{0} \leq 0$.

In contrast, away from instability ( $\omega$ well below $\omega_{2}$ ), quantum effects become much smaller even though they remain non-zero, as seen in Fig. 2 for $\omega=0.15 \omega_{1}$ (where $\left.\omega_{+} \approx 1.04 \omega_{1}, \omega_{-} \approx 0.45 \omega_{1}\right)$. Alternating squeezing in both $P_{j}$ and $Q_{j}$ is now observed (the behavior of $\Delta P_{2}$ and $\Delta Q_{2}$ is analogous) and the correspondence with the evolution of entanglement (i.e. with $f(t)$ ) is less direct, with the maxima of $f(t)$ reflecting essentially the largest squeezing (that of $Q_{1}$ or $P_{2}$ ). Nonetheless, the average boson numbers $\left\langle N_{j}(t)\right\rangle$ follow approximately $f(t)$.

We can easily understand these results by considering the expansion of the exact expressions (36) - (41) for small $\omega\left(|\omega| \ll \operatorname{Min}\left[\omega_{1}, \omega_{2}\right]\right)$. We obtain, neglecting terms of 
Exact dynamics and squeezing in two harmonic modes coupled through angular momentum11
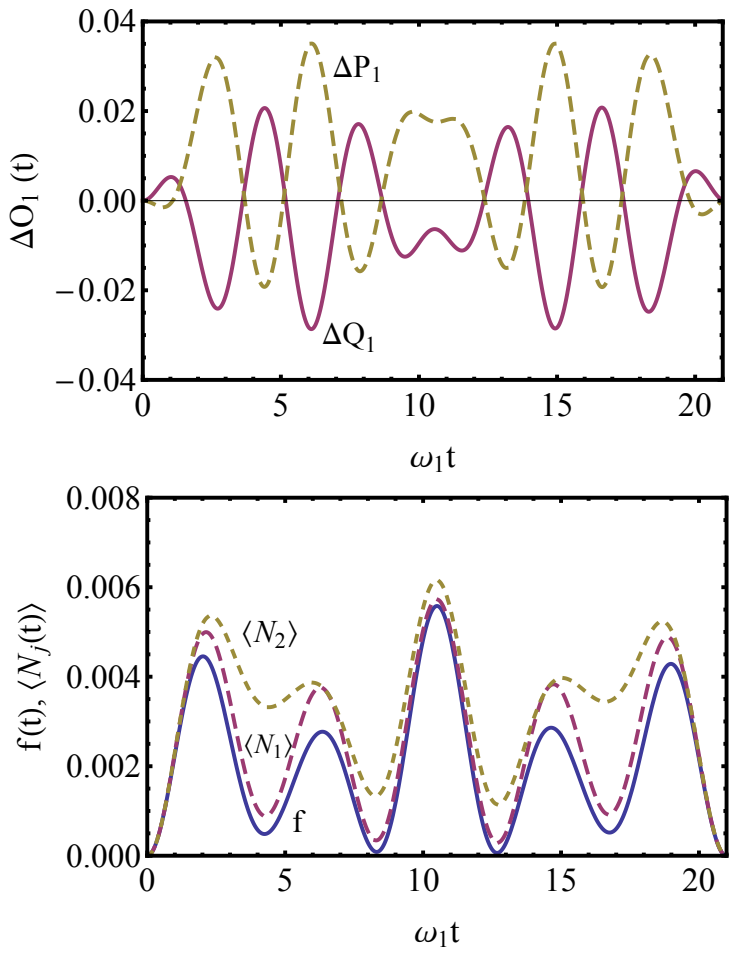

Figure 2. (Color online) Top: The shifted squeezing ratios for $Q_{1}$ and $P_{1}$ for weak coupling $\omega=0.15 \omega_{1}$. Now both $Q_{j}$ and $P_{j}$ exhibit small alternating squeezing. Bottom: The corresponding value of $f(t)$ (43), together with the average boson number of each oscillator. The behavior of the entanglement entropy $S(t)$ (42) is similar to that of $f(t)$.

order $\omega^{4}$

$$
\begin{aligned}
\left\langle N_{j}(t)\right\rangle_{0} & \approx \frac{\omega^{2}\left(\omega_{1}-\omega_{2}\right)^{2}}{\omega_{1} \omega_{2}\left(\omega_{1}+\omega_{2}\right)^{2}} \sin ^{2} \frac{\omega_{1}+\omega_{2}}{2} t \\
\left\langle a_{j}^{2}(t)\right\rangle_{0} & \approx i(-1)^{j+1} \frac{\omega^{2}\left(\omega_{1}^{2}-\omega_{2}^{2}\right)}{2 \omega_{1} \omega_{2} \omega_{j}} e^{-i \frac{\omega_{1}+\omega_{2}}{2} t}\left[e^{\left.-i \omega_{j} t \frac{\sin \frac{\omega_{1}-\omega_{2}}{2} t}{\omega_{1}-\omega_{2}}-\frac{\sin \frac{\omega_{1}+\omega_{2}}{2} t}{\omega_{1}+\omega_{2}}\right]},\right.
\end{aligned}
$$

which show that both $\left\langle N_{j}(t)\right\rangle_{0}$ and $\left\langle a_{j}^{2}(t)\right\rangle_{0}$ are of order $\omega^{2}$, with $\left\langle N_{1}(t)\right\rangle_{0}=\left\langle N_{2}(t)\right\rangle_{0}$ at this order. This implies that $\left|\left\langle a_{j}^{2}(t)\right\rangle_{0}\right|^{2}$ will be of order $\omega^{4}$, so that Eq. (43) leads to $f(t) \approx\left\langle N_{j}(t)\right\rangle_{0}$ up to $O\left(\omega^{2}\right)$. Hence, the $\left\langle N_{j}(t)\right\rangle_{0}$ and $f(t)$ will be close for small $\omega$.

Besides, $\operatorname{Re}\left[\left\langle a_{j}^{2}(t)\right\rangle_{0}\right]$ will change its sign as $t$ evolves, indicating that squeezing will alternate between $Q_{j}$ and $P_{j}$, being again larger for the oscillator with the lowest frequency due to the factor $\omega_{j}^{-1}$ in (48). Note that for small $\left\langle N_{j}(t)\right\rangle_{0}$ and $\left\langle a_{j}(t)\right\rangle_{0}$, $R_{Q_{j}\left(P_{j}\right)}(t) \approx 1+\left\langle N_{j}(t)\right\rangle_{0} \pm \operatorname{Re}\left[\left\langle a_{j}(t)^{2}\right\rangle_{0}\right]$, which will not strictly follow $\left\langle N_{j}(t)\right\rangle_{0}$, as $\left\langle a_{j}^{2}(t)\right\rangle_{0}$ is of the same order as $\left\langle N_{j}^{2}(t)\right\rangle_{0}$ but not proportional to it. Finally, it is verified from (47)-(48) that $\left\langle N_{j}(t)\right\rangle_{0}$ and $\left\langle a_{j}^{2}(t)\right\rangle_{0}$ are again proportional to $\left(\omega_{1}-\omega_{2}\right)^{2}$ and $\omega_{1}^{2}-\omega_{2}^{2}$ respectively, hence vanishing for $\omega_{1}=\omega_{2}$ and leading to a larger entanglement and squeezing as the anisotropy $\omega_{1}-\omega_{2}$ increases.

We finally mention that for short times $t$ such that $\omega_{ \pm} t \ll 1$, we obtain, after an 
Exact dynamics and squeezing in two harmonic modes coupled through angular momentum 12 expansion up to $O\left(t^{2}\right)$ of the exact expressions (36)-(41),

$$
\left\langle N_{j}(t)\right\rangle_{0} \approx \frac{\omega^{2}\left(\omega_{1}-\omega_{2}\right)^{2} t^{2}}{4 \omega_{1} \omega_{2}},\left\langle a_{j}^{2}(t)\right\rangle_{0} \approx(-1)^{j+1} \frac{\omega^{2}\left(\omega_{1}^{2}-\omega_{2}^{2}\right) t^{2}}{4 \omega_{1} \omega_{2}},
$$

which are in agreement with the $t \rightarrow 0$ limits of Eqs. (47)-(48). Hence, these quantities increase initially quadratically with time $t$, with $\left\langle N_{1}(t)\right\rangle_{0}=\left\langle N_{2}(t)\right\rangle_{0} \approx f(t)$ in this limit. Moreover, in this regime $\left\langle a_{1}^{2}(t)\right\rangle_{0}=-\left\langle a_{2}^{2}(t)\right\rangle_{0}$ is real, and positive if $\omega_{1}>\omega_{2}$. This entails that squeezing will initially start in $P_{1}$ (as seen in Fig. 2) and $Q_{2}$, with

$$
R_{P_{1}}(t) \approx R_{Q_{2}}(t) \approx 1-\frac{\omega^{2}\left(\omega_{1}-\omega_{2}\right) \omega_{2}}{2 \omega_{1} \omega_{2}} t^{2}
$$

\section{Conclusions}

We have derived the exact analytical closed form solution for the field operators of two linear oscillators coupled through angular momentum. We then applied the solution to investigate the relation between squeezing and entanglement generation in this model when starting from a separable coherent state. In the vicinity of instability, the generated entanglement between the modes shows a large amplitude-low frequency behavior (almost periodic), which is reflected in a similar behavior of the squeezing in the coordinate of one of modes and the momentum of the other mode. A different behavior occurs in the weak coupling regime, away from instability, where the generated entanglement is small and the squeezing is weak, exhibiting an essentially alternating behavior for the coordinate and momentum of each oscillator. Approximate analytical expressions describing these two regimes have also been derived from the general exact solution.

The present solution has of course potential applications for studies of other quantum statistical properties such as higher order squeezing, antibunching of photons and other nonclassical photon statistics. The solution is also of interest for quantum information applications. As stated in the introduction, the present model admits distinct physical realizations, so that results could in principle be tested in quite different scenarios (optical simulations, particles in anisotropic harmonic traps, condensates, etc.). We remark, finally, that expressions similar to (10)-(11) and (36), (41) remain formally valid for general systems of $n$ harmonic modes interacting through quadratic (in $a_{j}, a_{j}^{\dagger}$ ) couplings, replacing $\mathcal{H}$ by the corresponding $2 n \times 2 n$ matrix.

Acknowledgements: We are thankful to the Third World Academy of Sciences (TWAS), Trieste, Italy and CONICET of Argentina, for financial support through TWAS-UNESCO fellowship program. NC and RR acknowledge support from CONICET and CIC of Argentina, while SM thanks the University Grants Commission, Government of India, for support through the research project (F.No.42-852/2013(SR)). 


\section{Appendix}

By means of the canonical transformation $(j=1,2)$

$$
P_{j}^{\prime}=P_{j}+\gamma Q_{3-j}, Q_{j}^{\prime}=\left(Q_{j}-\eta P_{3-j}\right) /(1+\eta \gamma)
$$

where $\gamma=\frac{2 \Delta-\omega_{1}^{2}+\omega_{2}^{2}}{4 \omega}, \eta=\frac{2 \gamma}{\omega_{1}^{2}+\omega_{2}^{2}}$, we may rewrite (1) in the decoupled form (we set here $m=1)$

$$
H=\frac{1}{2} \sum_{j=1,2}\left(\alpha_{j}{P_{j}^{\prime}}^{2}+\beta_{j}{Q_{j}^{\prime}}^{2}\right),
$$

where $\alpha_{j}=1-\frac{\omega}{\Delta}\left(\gamma+(-1)^{j} \omega\right), \beta_{j}=\frac{\Delta}{\omega}\left(\gamma-(-1)^{j} \omega\right)$. Here $\alpha_{j}>0, \beta_{j}>0$ for $j=1,2$ in the fully stable region $\omega<\omega_{2}$, whereas $\alpha_{1}>0, \beta_{1}>0, \alpha_{2}<0, \beta_{2}<0$ in the second dynamically stable sector $\omega>\omega_{1}$, with $\alpha_{2}>0, \beta_{2}<0$ in the unstable sector $\omega_{2}<\omega<\omega_{1}$ and $\beta_{2}=0$ at the borders $\omega=\omega_{2}$ or $\omega=\omega_{1}$. Eq. (52) then leads, in the dynamically stable regions $\omega<\omega_{2}$ or $\omega>\omega_{1}$, to

$$
H=\hbar \omega_{+} \frac{P_{+}^{2}+Q_{+}^{2}}{2} \pm \hbar \omega_{-} \frac{P_{-}^{2}+Q_{-}^{2}}{2}
$$

where $\omega_{ \pm}=\sqrt{\alpha_{1} \beta_{1}}$ (real), $P_{ \pm}=\sqrt[4]{\alpha_{2} / \hbar \beta_{1}} P_{\frac{1}{2}}, Q_{ \pm}=\sqrt[4]{\beta_{\frac{1}{2}} / \hbar \alpha_{1}} Q_{\frac{1}{2}}$, and the minus sign in (53) applies for $\omega>\omega_{1}$. Eqs. (33)-(34) are then obviously obtained for $b_{ \pm}=\left(Q_{ \pm}+i P_{ \pm}\right) / \sqrt{2}, b_{ \pm}^{\dagger}=\left(Q_{ \pm}-i P_{ \pm}\right) / \sqrt{2}$. The possible normal representations in the unstable regime are discussed in detail in [23, 41].

\section{References}

[1] Louisell W H 1990 Quantum Statistical Properties of Radiation, Wiley (NY)

[2] Walls D F and Milburn G J 1994 Quantum Optics, Springer (Berlin)

[3] Mollow B R Phys. Rev. 19671621256

[4] Estes L E, Keil T H and Narducci L M 1968 Phys. Rev. 175286

[5] Iafrate G J and Croft M 1975 Phys. Rev. A 121525

[6] Holzwarth G and Chabay I 1972 J. Chem. Phys. 571632

[7] Belkin M A, Shen Y R and Flytzanis C 2002 Chem. Phys. Lett. 363479

[8] Cochrane P T, Milburn G J and Munro W J 2000 Phys. Rev. A 62062307

[9] Fan H, Li C and Jiang Z 2004 Phys. Lett. A 327416

[10] Ng K M, Lo C F 1997 Phys. Lett. A 230144

[11] Valatin J G 1956 Proc. R. Soc. London 238132

[12] Feldman A and Kahn A H 1970 Phys. Rev. B 14584

[13] Ring P and Schuck P 1980 The Nuclear Many-Body Problem, Springer (NY); Blaizot J P Ripka G 1986 Quantum Theory of Finite Systems, MIT Press (MA)

[14] Madhav A V and Chakraborty T 1994 Phys. Rev. B 49 (1994) 8163

[15] Linn M, Niemeyer M and Fetter A L 2001 Phys. Rev. A 64023602

[16] Oktel M Ö 2004 Phys. Rev. A 69023618

[17] Fetter A L 2007 Phys. Rev. A 75013620

[18] Aftalion A, Blanc X and Lerner N 2009 Phys. Rev. A 79 011603(R)

[19] Aftalion A, Blanc X and Dalibard J 2005 Phys. Rev. A 71023611

[20] Bloch I, Dalibard J and Zwerger W 2008 Rev. Mod. Phys. 80885

[21] Fetter A L 2009 Rev. Mod. Phys. 81647 
Exact dynamics and squeezing in two harmonic modes coupled through angular momentum 14

[22] Pěrina J, Hradil Z and Jurčo B 1994 Quantum Optics and Fundamentals of Physics, Kluwer, Dordrecht

[23] Rossignoli R and Kowalski A M 2009 Phys. Rev. A 79062103

[24] Rebón L and Rossignoli R 2011 Phys. Rev. A 84052320

[25] Rebón L, Canosa N and Rossignoli R 2014 Phys. Rev. A 89042312

[26] Schachenmayer J et al 2013 Phys. Rev. X 3 031015; Daley A J et al 2012 Phys. Rev. Lett. 109 020505; Bardarson J H, Pollmann F and Moore J E 2012 Phys. Rev. Lett. 109017202

[27] Nielsen M and Chuang I L 2000 Quantum Computation and Quantum Information (Cambridge Univ. Press, Cambridge, UK)

[28] Sørensen A et al 2001 Nature 409 63; Orzel C et al 2001 Science 291 2386; Bigelow N 2001 Nature 40927

[29] Esteve J et al 2008 Nature 4551216

[30] Gühne O and Toth G 2009 Phys. Rep. 4741

[31] Ma J, Wang X, Sun C P, Nori F 2011 Phys. Rep. 50989

[32] Choi S and Bigelow N P 2005 Phys. Rev. A 72033612

[33] Toth G, Knapp C, Gühne O and Briegel H J, 2009 Phys. Rev. A 79042334.

[34] Chung N N et al 2010 Phys. Rev. A 82 014101; Chew L C and Chung N N 2014 Symmetry 6295

[35] Gross C 2012 J. Phys. B: At. Mol. Phys. 45103001

[36] Sen B and Mandal S 2005 J. Mod. Opt 52 1789; Sen B, Mandal S and Pěrina J 2007 J. Phys. B: At. Mol. Phys. 401417

[37] Sen B et al 2013 Phys. Rev. A 87022325

[38] Audenaert K et al 2006 Phys. Rev. A 66042327

[39] Adesso G, Serafini A and Illuminati F 2004 Phys. Rev. A 70, 022318; Serafini A, Adesso G and Illuminati F 2005 Phys. Rev. A 71032349

[40] Braunstein S L and van Loock P 2005 Rev. Mod. Phys. 77 513; Weedbrook C et al 2012 Rev. Mod. Phys. 84621

[41] Rossignoli R and Kowalski A M 2005 Phys. Rev. A 72032101 